\title{
The scalar radius of the pion in two-flavor Wilson lattice QCD
}

\author{
Vera Gülpers ${ }^{* 1,2}$, Georg von Hippel ${ }^{1}$, Hartmut Wittig ${ }^{1,2}$ \\ ${ }^{1}$ PRISMA Cluster of Excellence, Institut für Kernphysik, Johannes Gutenberg Universität Mainz, \\ 55099 Mainz, Germany \\ ${ }^{2}$ Helmholtz Institute Mainz, Johannes Gutenberg Universität Mainz, 55099 Mainz, Germany \\ E-mail: guelpers@kph.uni-mainz.de
}

\begin{abstract}
We present a calculation of the scalar charge radius of the pion using $N_{f}=2$ dynamical flavors of non-perturbatively $O(a)$-improved Wilson fermions, extending the calculation to a wider range of pion masses for a fixed lattice spacing a. We find that the disconnected contribution to the scalar radius is not negligible especially for smaller pion masses, and is required in order to obtain the behavior expected from next-to-leading order (NLO) Chiral Perturbation Theory ( $\chi$ PT). The low-energy constant $\bar{\ell}_{4}$ is determined from a fit to NLO $\chi$ PT.
\end{abstract}

31st International Symposium on Lattice Field Theory LATTICE 2013

July 29 - August 3, 2013

Mainz, Germany

* Speaker. 


\section{Introduction}

The $Q^{2}$-dependence of the scalar pion form factor

$$
F_{\mathrm{S}}^{\pi}\left(Q^{2}\right) \equiv\left\langle\pi^{+}\left(p_{f}\right)\left|m_{\mathrm{d}} \bar{d} d+m_{\mathrm{u}} \bar{u} u\right| \pi^{+}\left(p_{i}\right)\right\rangle \quad \text { with } Q^{2}=-\left(p_{f}-p_{i}\right)^{2}
$$

at vanishing momentum transfer determines the scalar radius $\left\langle r^{2}\right\rangle_{\mathrm{s}}^{\pi}$ of the pion. Since at next-toleading order in $\chi \mathrm{PT}$ it depends only on the low-energy constant $\ell_{4}$ [1], a calculation of $\left\langle r^{2}\right\rangle_{\mathrm{S}}^{\pi}$ provides a possibility to determine $\ell_{4}$ independently from other low-energy constants.

The matrix element (1.1) receives a connected and a disconnected contribution (cf. figure 1). The computation of the latter requires knowledge of the all-to-all propagator, which we calculate using stochastic sources and a generalized hopping parameter expansion as explained in section 3. The scalar form factor is extracted from three- and two-point functions by building appropriate ratios.For more details on this work, the reader is referred to our recent paper [2].

\section{Simulation Setup}

For our calculation we use $N_{f}=2$ dynamical flavors of $\mathscr{O}(a)$-improved Wilson fermions. The corresponding Wilson-Dirac operator has the form

$$
D_{S W}=\frac{1}{2 \kappa} \mathbb{1}-\frac{1}{2} H+c_{S W} B,
$$

with the $\mathscr{O}(a)$-improvement term $c_{S W} B$ and the hopping matrix $H$.

Our simulations are performed using gauge ensembles produced as part of the CLS initiative. An overview of the ensembles used in this study is given in table 1 . So far only a single value of the gauge coupling $\beta=5.3$ has been considered with a corresponding lattice spacing of $a=0.063 \mathrm{fm}$ [3]. Since all ensembles fulfill $m_{\pi} L \geq 4$, we assume finite size effects to be negligible.

\begin{tabular}{cccccccc}
\hline$\beta$ & $a[\mathrm{fm}]$ & lattice & $m_{\pi}[\mathrm{MeV}]$ & $m_{\pi} L$ & $\kappa$ & Label & Statistics \\
\hline \hline 5.3 & 0.063 & $64 \times 32^{3}$ & 650 & 6.6 & 0.13605 & E3 & 156 \\
5.3 & 0.063 & $64 \times 32^{3}$ & 605 & 6.2 & 0.13610 & E4 & 162 \\
5.3 & 0.063 & $64 \times 32^{3}$ & 455 & 4.7 & 0.13625 & E5 & 1000 \\
\hline 5.3 & 0.063 & $96 \times 48^{3}$ & 325 & 5.0 & 0.13635 & F6 & 300 \\
5.3 & 0.063 & $96 \times 48^{3}$ & 280 & 4.3 & 0.13638 & F7 & 351 \\
\hline
\end{tabular}

Table 1: Overview of the CLS ensembles used in this work

\section{Calculation of the disconnected loop}

For the calculation of the disconnected loop contained in the scalar pion form factor, the allto-all propagator is needed. Usually one uses stochastic sources [4] to invert the Dirac-operator,

$$
D_{S W}^{-1}=\frac{1}{N} \sum_{i}\left|s_{i}\right\rangle\left\langle\eta_{i}\right| \quad \text { with } \quad D_{S W}\left|s_{i}\right\rangle=\left|\eta_{i}\right\rangle .
$$




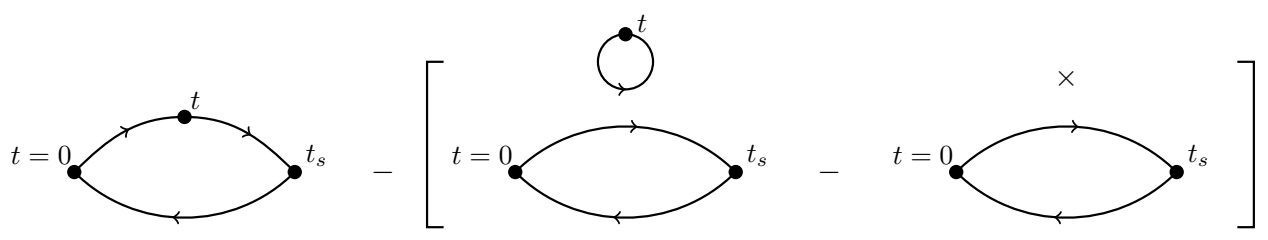

Figure 1: The connected and the disconnected three-point function with subtracted vacuum

To limit the computational overhead, the number $N$ of stochastic sources used in the calculation has to be small. However, when using fewer sources, the stochastic error, which is expected to be proportional to $1 / \sqrt{N}$, gets larger. Previous studies [2,4] have shown that a generalized hopping parameter expansion (HPE) is a powerful tool to improve the stochastic estimate of the propagator for a given number $N$ of stochastic sources. With the HPE the inverse of the $\mathscr{O}(a)$-improved Wilson-Dirac operator can be calculated as

$$
D_{S W}^{-1}=\sum_{i=0}^{k-1}\left(\frac{1}{2} A^{-1} H\right)^{i} A^{-1}+\left(\frac{1}{2} A^{-1} H\right)^{k} D_{S W}^{-1}
$$

where the inverse $D_{S W}^{-1}$ on the right hand side of (3.2) can be calculated with stochastic sources (c.f (3.1)). In (3.2) the inverse of the matrix $A=(2 \kappa)^{-1} \mathbb{1}+c_{S W} B$ is needed, which due to the locality of the $\mathscr{O}(a)$-improvement term requires inverting two $6 \times 6$-matrices for every lattice point, which is comparatively cheap in computer time.

For the calculation of the disconnected loop we use $N=3$ stochastic sources for the inversion and $k=6$ terms in the generalized hopping parameter expansion.

\section{The scalar form factor}

\subsection{Extracting the form factor}

Neglecting exited states, the two-point function of the pseudoscalar density $\phi(x)=\bar{q}(x) \gamma_{5} q(x)$ is expected to behave like

$$
C_{2 \mathrm{pt}}\left(t_{s}, \mathbf{p}\right)=\sum_{\mathbf{x}} e^{-i \mathbf{p} \cdot \mathbf{x}}\left\langle\phi\left(t_{s}, \mathbf{x}\right) \phi(0)\right\rangle \sim \frac{Z_{\mathbf{p}}^{2}}{2 E_{\mathbf{p}}}\left[e^{-t_{s} E_{\mathbf{p}}}+e^{-\left(T-t_{s}\right) E_{\mathbf{p}}}\right],
$$

where $Z_{\mathbf{p}}^{2}=|\langle\pi(\mathbf{p})|\phi(0)| 0\rangle|^{2}$ is the probability for $\phi$ for creating a pion of momentum $\mathbf{p}$.

Inserting the scalar density $\mathscr{O}_{\mathbf{S}}(y)=\bar{q}(y) q(y)$ at a time $t$ with $0<t<t_{S}$, one obtains the threepoint function

$$
\begin{aligned}
C_{3 \mathrm{pt}}\left(t, t_{s}, \mathbf{p}_{i}, \mathbf{p}_{f}\right) & =\sum_{\mathbf{x}, \mathbf{y}} e^{-i \mathbf{p}_{f} \cdot \mathbf{x}+i \mathbf{q} \cdot \mathbf{y}}\left\langle\phi\left(t_{s}, \mathbf{x}\right) \mathscr{O}(t, \mathbf{y}) \phi(0)\right\rangle \\
& \sim \frac{Z_{\mathbf{p}_{i}} Z_{\mathbf{p}_{f}}}{4 E_{\mathbf{p}_{i}} E_{\mathbf{p}_{f}}}\left\langle\pi\left(\mathbf{p}_{f}\right)\left|\mathscr{O}_{\mathbf{S}}(0)\right| \pi\left(\mathbf{p}_{i}\right)\right\rangle e^{-\left(t_{s}-t\right) E_{\mathbf{p}_{f}}} e^{-t E_{\mathbf{p}_{i}}}
\end{aligned}
$$

with the scalar pion form factor $\left\langle\pi\left(\mathbf{p}_{f}\right)\left|\mathscr{O}_{\mathbf{S}}(0)\right| \pi\left(\mathbf{p}_{i}\right)\right\rangle$. Since the vacuum contribution of the scalar density,

$$
C_{\mathrm{vac}}\left(t, t_{s}, \mathbf{p}_{i}, \mathbf{p}_{f}\right)=C_{2 \mathrm{pt}}\left(t_{s}, \mathbf{p}_{f}\right) \sum_{\mathbf{y}} e^{i \mathbf{q} \cdot \mathbf{y}}\left\langle\mathscr{O}_{\mathrm{S}}(t, \mathbf{y})\right\rangle,
$$


is non-zero, it has to be subtracted before equation (4.2) can be employed to determine the scalar form factor. Figure 1 shows the three contributions to the three-point function, i.e. the connected and the disconnected contributions with subtracted vacuum. Note that the vacuum subtraction also cancels the additive renormalization $b_{0}$ of the scalar density

$$
\left\langle\mathscr{O}^{R}\right\rangle=Z_{s}\left\langle\mathscr{O}-b_{0}\right\rangle,
$$

which is a consequence of the breaking of the chiral symmetry by the Wilson term. The remaining multiplicative renormalization constant $Z_{s}$ is not determined in this work, since it cancels in the calculation of the scalar radius.

To extract the scalar form factor from (4.2) it is convenient to build ratios of three- and twopoint functions [5]. In this work two different ratios

$$
\begin{aligned}
& R_{1}\left(t, t_{s}, \mathbf{p}_{i}, \mathbf{p}_{f}\right)=\sqrt{\frac{C_{3 \mathrm{pt}}\left(t, t_{s}, \mathbf{p}_{i}, \mathbf{p}_{f}\right) C_{3 \mathrm{pt}}\left(t, t_{s}, \mathbf{p}_{f}, \mathbf{p}_{i}\right)}{C_{2 \mathrm{pt}}\left(t_{s}, \mathbf{p}_{i}\right) C_{2 \mathrm{pt}}\left(t_{s}, \mathbf{p}_{f}\right)}} \\
& R_{3}\left(t, t_{s}, \mathbf{p}_{i}, \mathbf{p}_{f}\right)=\frac{C_{3 \mathrm{pt}}\left(t, t_{s}, \mathbf{p}_{i}, \mathbf{p}_{f}\right)}{C_{2 \mathrm{pt}}\left(t_{s}, \mathbf{p}_{f}\right)} \sqrt{\frac{C_{2 \mathrm{pt}}\left(t_{s}, \mathbf{p}_{f}\right) C_{2 \mathrm{pt}}\left(t, \mathbf{p}_{f}\right) C_{2 \mathrm{pt}}\left(t_{s}-t, \mathbf{p}_{i}\right)}{C_{2 \mathrm{pt}}\left(t_{s}, \mathbf{p}_{i}\right) C_{2 \mathrm{pt}}\left(t, \mathbf{p}_{i}\right) C_{2 \mathrm{pt}}\left(t_{s}-t, \mathbf{p}_{f}\right)}}
\end{aligned}
$$

will be used. Inserting the expressions for three- and two-point functions in $R_{1}$ (4.5) one obtains

$$
R_{1}\left(t, t_{s}, \mathbf{p}_{i}, \mathbf{p}_{f}\right) \sim \frac{\left\langle\pi\left(\mathbf{p}_{f}\right)\left|\mathscr{O}_{\mathrm{S}}\right| \pi\left(\mathbf{p}_{i}\right)\right\rangle}{2 \sqrt{E_{\mathbf{p}_{i}} E_{\mathbf{p}_{f}}}} \sqrt{\frac{e^{-E_{\mathbf{p}_{i}} t_{s}} e^{-E_{\mathbf{p}_{f}} t_{s}}}{\left(e^{-E_{\mathbf{p}_{i}} t_{s}}+e^{-E_{\mathbf{p}_{i}}\left(T-t_{s}\right)}\right) \cdot\left(e^{-E_{\mathbf{p}_{f}} t_{s}}+e^{-E_{\mathbf{p}_{f}}\left(T-t_{s}\right)}\right)}},
$$

where all the overlap factors $Z_{\mathbf{p}}$ and the dependence of the operator insertion time $t$ are canceled. The pion energies $E_{\mathbf{p}}$ in the remaining $t_{s}$-dependent part, can be extracted from two-point functions.

For $R_{3}$ one obtains

$$
R_{3}\left(t, t_{s}, \mathbf{p}_{i}, \mathbf{p}_{f}\right) \sim \frac{\left\langle\pi\left(\mathbf{p}_{f}\right)\left|\mathscr{O}_{\mathrm{S}}\right| \pi\left(\mathbf{p}_{i}\right)\right\rangle}{2 \sqrt{E_{\mathbf{p}_{i}} E_{\mathbf{p}_{f}}}} f\left(t, t_{s}\right)
$$

when two- and three-point functions are inserted. The left-over time-dependence $f\left(t, t_{s}\right)$ is analytically known and again contains only pion energies $E_{\mathbf{p}}$. For large time separations $0 \ll t \ll t_{s} \ll T / 2$ the time-dependence vanishes, i.e. $f\left(t, t_{s}\right) \rightarrow 1$. Note that, in contrast to (4.7), equation (4.8) is only valid if the same type of source is used at pion source and pion sink, e.g. local-local or smeared-smeared, since the overlap factors $Z_{\mathbf{p}}$ depend on the source type.

\subsection{Results}

Our results for the ratios obtained on the E5 ensemble (cf. table 1) are shown in figures 2 and 3 for $Q^{2}=0$ and $Q^{2}=0.278 \mathrm{GeV}^{2}$, respectively. Momenta have been inserted via Fourier transformation.

For the connected part of the scalar form factor we currently have only data from correlation functions with a local operator at the pion sink and Gaussian smearing at the pion source. Therefore, only ratio $R_{1}$ can be used. For the disconnected contribution to the scalar form factor we can make use of smeared-smeared two-point functions, thus both ratios can be applied. Since $R_{3}$ gives a much clearer signal for the disconnected part, we use $R_{3}$ for the analysis. 

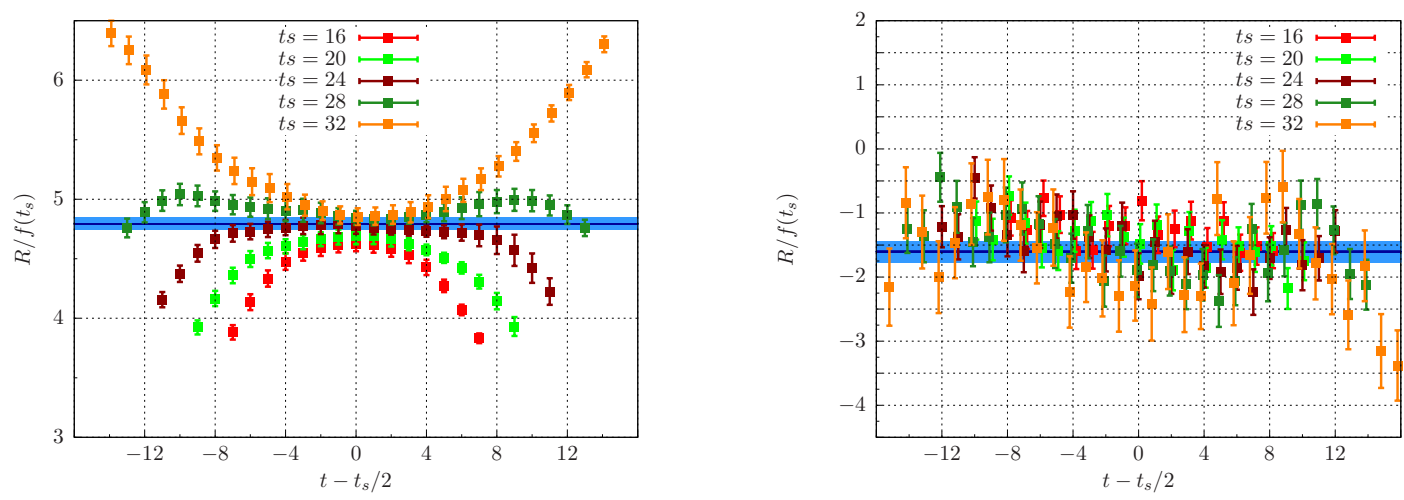

Figure 2: Our results for the ratios for $Q^{2}=0 \mathrm{GeV}^{2}$. The connected contribution is shown on the left, the disconnected on the right. In both cases the ratios are divided by the time-dependence.
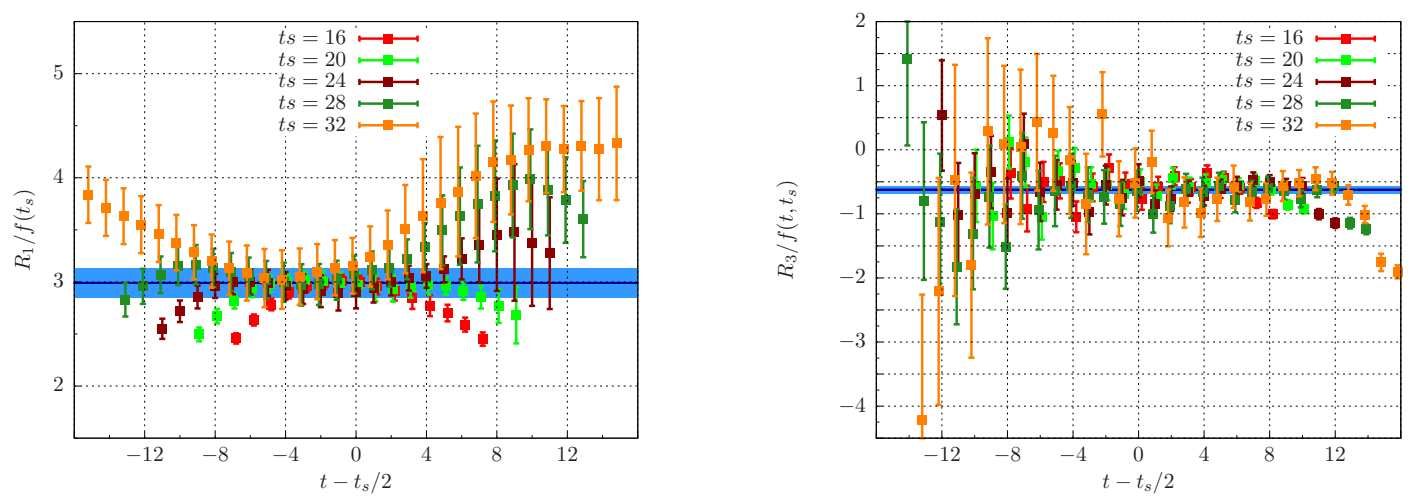

Figure 3: Our results for the ratios for $Q^{2}=0.278 \mathrm{GeV}^{2}$. We use $R_{1}$ for the connected contribution (right) and $R_{3}$ for the disconnected contribution (left). In both cases the ratios are divided by the appropriate timedependence.

In all data sets the ratios are divided by the known time-dependences (cf. (4.7) and (4.8)), such that a plateau independent in $t_{s}$ can be expected. Nevertheless, we observe that for $Q^{2}=0$ the plateau values grow as $t_{s}$ is increased, an effect which we attribute to excited state contaminations. To avoid systematics from excited states, ratios with $t_{s}<24$ have been omitted from the analysis. The blue lines in figures 2 and 3 show the results of a global fit to the plateau regions of the data with $t_{s} \geq 24$.

\section{The Scalar Radius}

The scalar radius is defined as

$$
\left\langle r^{2}\right\rangle_{\mathrm{s}}^{\pi}=-\left.\frac{6}{F_{\mathrm{s}}^{\pi}(0)} \frac{\partial F_{\mathrm{s}}^{\pi}\left(Q^{2}\right)}{\partial Q^{2}}\right|_{Q^{2}=0} .
$$

So far, we have results for $Q^{2}=0$ and the smallest non-vanishing momentum transfer. For higher momenta the ratios get very noisy and the signal is essentially lost. However, we can obtain an estimate for the scalar radius from two different momentum transfers using a parameterization linear 


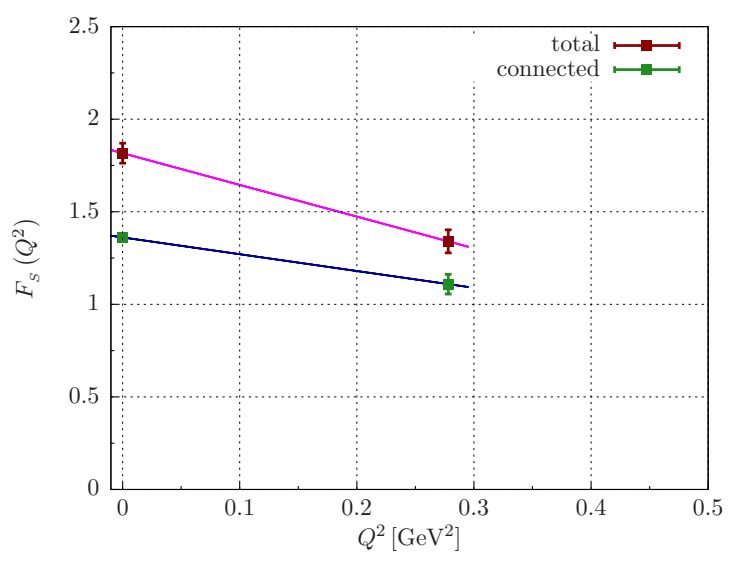

Figure 4: The $Q^{2}$-dependence of the scalar form factor. The red points are our results for the total form factor, the green points for the connected contribution only. In both cases a linear curve was matched to the data to estimate the scalar radius.

in $Q^{2}$. Figure 4 shows our results for the scalar form factor plotted against the momentum transfer for the E5 ensemble. The red points are the values for the total form factor, i.e. connected and disconnected, the green points are the results we obtain neglecting the disconnected contribution. To estimate the scalar radius a linear curve was matched to the data.

In figure 5 our results for the scalar radius for the different ensembles are plotted against the pion mass $m_{\pi}^{2}$. The red points are the data from the total form factor, whereas the yellow points show the results from the connected contribution only. Clearly, the disconnected contribution to the scalar radius is not negligible, in qualitative agreement with results from partially quenched $\chi \mathrm{PT}$ [6].

To extrapolate to the physical pion mass, we use chiral perturbation theory. At next-to-leading order, the scalar radius is given by [1]

$$
\left\langle r^{2}\right\rangle_{\mathrm{s}}^{\pi}=\frac{1}{(4 \pi F)^{2}}\left(-\frac{13}{2}\right)+\frac{6}{(4 \pi F)^{2}}\left[\bar{\ell}_{4}+\ln \left(\frac{m_{\pi, p h y s}^{2}}{m_{\pi}^{2}}\right)\right],
$$

and depends only on one low-energy constant $\bar{\ell}_{4}$. The blue line in figure 5 shows the result of a fit of (5.2) to our data, where $\bar{\ell}_{4}$ is used as a free fit parameter. For the scalar radius at physical pion mass we obtain $\left\langle r^{2}\right\rangle_{\mathrm{s}}^{\pi}=0.633 \pm 0.021 \mathrm{fm}^{2}$, which is in good agreement with the value obtained from $\pi \pi$-scattering [7] shown in green in figure (5). Furthermore, from the fit we obtain $\bar{\ell}_{4}=4.73 \pm 0.12$, which agrees well with the result from the vector form factor [8] computed on the CLS ensembles. Note, that all errors are purely statistical, since no sources of systematic errors have been studied so far.

\section{Summary and Outlook}

Using stochastic sources and a generalized hopping parameter expansion, we are able to calculate the disconnected contribution to the scalar pion form factor precisely. When calculating the scalar radius, we find that the disconnected contribution is not negligible and is required to obtain 


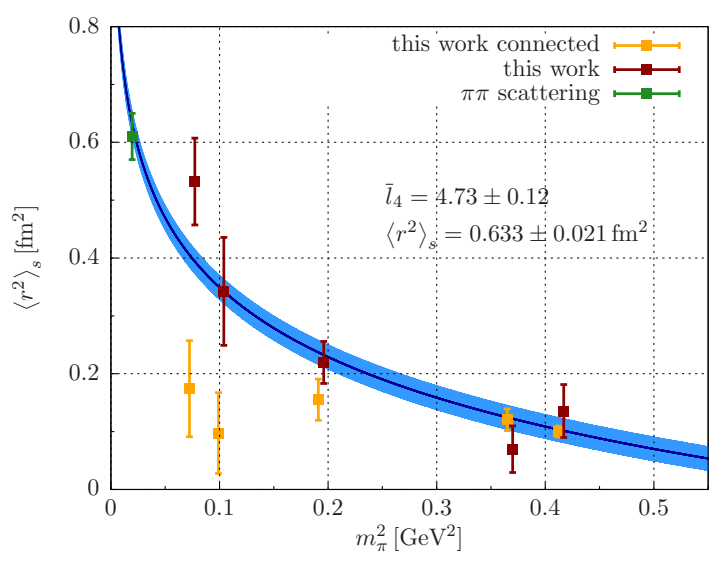

Figure 5: The $m_{\pi}^{2}$-dependence of the scalar radius. The red points show our results for the total form factor, the yellow points the results for the disconnected contribution only. The blue curve is a fit of NLO $\chi$ PT to the red points.

the behavior in $m_{\pi}^{2}$ as expected from NLO $\chi$ PT. A fit to our results enables us to determine the low-energy constant $\bar{\ell}_{4}$. The extrapolated value for the scalar radius at physical pion mass is in good agreement with the value from $\pi \pi$-scattering data. So far, no systematic errors have been determined and thus all errors quoted are purely statistical. In the future we plan to address different sources of stochastic errors, such as lattice artifacts or finite volume effects. Furthermore, we will extended our calculations to different lattice spacings, such that a continuum extrapolation can be performed.

Acknowledgements We acknowledge useful discussions with Andreas Jüttner, Bastian Brandt and Harvey B. Meyer. Our calculations were performed on the "Wilson" HPC Cluster at the Institute for Nuclear Physics, University of Mainz. We thank Dalibor Djukanovic and Christian Seiwerth for technical support. We are grateful for computer time allocated to project HMZ21 on the BlueGene computers "JUGENE" and "JUQUEEN" at NIC, Jülich. This research has been supported in part by the DFG in the SFB 1044. We are grateful to our colleagues in the CLS initiative for sharing ensembles.

\section{References}

[1] J. Gasser and H. Leutwyler, Ann. Phys. 158, (1984) 142-210

[2] V. Gülpers, G. von Hippel and H. Wittig, arXiv:1309.2104

[3] S. Capitani, M. Della Morte, G. von Hippel, B. Knippschild, H. Wittig, PoS LATTICE2011, 145 (2011), [arXiv:1110.6365]

[4] G. S. Bali, S. Collins and A. Schäfer, Comput. Phys. Commun. 181, (2010) 1570-1583, [arXiv:0910.2970]

[5] P. A. Boyle et al, JHEP 0705, (2007) 016, [hep-lat/0703005]

[6] A. Jüttner, JHEP 01, (2012) 007, [arXiv:1110.4859]

[7] G. Colangelo, J. Gasser and H. Leutwyler, Nucl. Phys. B603, (2001) 125-179, [hep-ph/0103088]

[8] B. B. Brandt, A. Jüttner and H. Wittig, arXiv:1306.2916 